\title{
Physical Separation of Motion into Three Categories for Evaluating the Behavioral Effects of Drugs in Mice
}

\section{Hiroto Shoji}

Department of Physics, Graduate School of Medical Science, Kyoto Prefectural University of Medicine, Taishogun, Kita-ku, Kyoto, Japan

\begin{abstract}
Characterization of open-field behavior and locomotor activity is widely used to assess the influence of a drug on animal behavior. In this study, we developed an index to characterize the behavior of drug-administered mice (C57BL/6). A three-exponential-model exhibited the best fit to the log-log plots of moving duration vs. frequency data of the different probability density functions. Therefore, as a heuristic argument, we divided each walking event into three categories according to the duration of movement. Then, we examined the number of walking events, and travel distances in each walking categories. Significant differences were observed more often when walking events were divided into the three categories. Therefore, this process would be useful to detect the effect of drugs on locomotor activity in mice.
\end{abstract}

Keywords: Locomotor activity; Exponential distribution; Cocaine; Alcohol; Nicotine

\section{Introduction}

Open-field and locomotor behaviors are commonly used to assess the influence of drugs on animal behavior [1]. A variety of methods to measure locomotive behavior have been previously developed. Photobeam interruptions or video tracking is used to measure the cumulative and general features of behavior, such as the distance covered by a mouse during an experimental session [2] or the ratio of the duration for which a mouse stays in the periphery of the setup arena to the duration for which it stays at the center $[3,4]$. The cumulative and general behaviors observed using these methods are believed to reflect the general view that open-field behavior is largely stochastic in nature and can be easily quantified by some measure of effects of drug [3].

Owing to the rapid advances in the development of software for visualization and analysis of open-field data measured automatically by video tracking, it is possible to obtain large amounts of data for analysis [5]. This allows for an open-ended approach in which new behavioral measures may be defined for specific effects on behavior [6-8]. Physicsoriented measures have been also developed for behavioral studies. Measures of locomotor activity derived from the scaling approach have been developed by Paulus et al. [9-11].

Interestingly, recent studies, suggest that the searching behavior of animals, including humans, can be characterized by power law or sum of exponential modes [12,13]. Moreover, how the characterized process is generated in Eschrichi coli has also been investigated using mathematical models [14].

In this paper, we described our further development of a method using the characterization described above. Focusing on the behavior effects of 3 drugs such as cocaine, ethanol and nicotine, we explored the statistical rule about moving duration. Since three-exponential model exhibited the best fit, we performed the division of walking event into three categories depending on the moving duration. To evaluate the division of walking events, we tested the traditional index to differentiate the behaviors in each divided category. Our main contribution lies in the construction of the basic strategy in which we assess the differences between processed with different time scales in such a way that the behavioral distinction are displayed more clearly.

\section{Materials and Methods}

\section{Animals}

Six-week-old male C57BL/6 (B6) mice were purchased from Shimizu Lab-oratory Supplies Co., Kyoto, Japan. The mice were housed in groups of 6 in standard breeding cages $(27 \times 21 \times 12 \mathrm{~cm})$ with freely available food and water under a 12-h light/12-h dark cycle (lights on at 08:00). The mice were acclimatized to these conditions for 2 weeks before the tests. The experiment was performed in accordance with the National Research Council's Guidelines for the Care and Use of Mammals in Neuroscience and Behavioral Research and with the approval of the Committee for Animal Research at Kyoto Prefectural University of Medicine.

\section{Preparation of drugs}

Cocaine (COC) (Takeda, Japan) was dissolved in a $0.9 \%$ saline (SA) solution $(0.9 \% \mathrm{NaCl})$ to $5 \mathrm{mg} / \mathrm{kg}$ COC (COC 5$), 10 \mathrm{mg} / \mathrm{kg} \mathrm{COC}$ (COC10), and $20 \mathrm{mg} / \mathrm{kg}$ COC (COC20). Ethanol (ETH) was prepared by diluting $95 \%$ with tap water to concentrations of $1.0 \mathrm{~g} / \mathrm{kg}$ (ETH1), $2.0 \mathrm{~g} / \mathrm{kg}$ (ETH2), and $4.0 \mathrm{~g} / \mathrm{kg}$ (ETH4). L-nicotine (NIC) solutions were prepared by diluting L-nicotine hemisulfate $(400 \mathrm{mg} / \mathrm{mL}$; Sigma) to concentrations of $2.0 \mathrm{~g} / \mathrm{mL}$ (NIC2), $4.0 \mathrm{~g} / \mathrm{mL}$ (NIC4), and $8.0 \mathrm{~g} / \mathrm{mL}$ (NIC8).

\section{Open field measurements}

The mice were tested once during the light phase of the photoperiodic cycle. From 7 9 days before the experiment, each mouse was handled daily for $3 \mathrm{~min}$ and exposed to the environment in which the observation was performed for the following $5 \mathrm{~min}$. This protocol was followed until the day of the observation.

*Corresponding author: Hiroto Shoji, Department of Physics, Graduate School of Medical Science, Kyoto Prefectural University of Medicine, Taishogun, Kita-ku, Kyoto, 603-8334, Japan, Tel: +81-75-465-7664, E-mail: shoji@koto.kpu-m.ac.jp

Received July 4, 2014; Accepted August 30, 2014; Published September 10, 2014

Citation: Shoji H (2014) Physical Separation of Motion into Three Categories for Evaluating the Behavioral Effects of Drugs in Mice. Int J Neurorehabilitation 1: 120 doi:10.4172/2376-0281.1000120

Copyright: @ 2014 Shoji $\mathrm{H}$. This is an open-access article distributed under the terms of the Creative Commons Attribution License, which permits unrestricted use, distribution, and reproduction in any medium, provided the original author and source are credited. 
On the day of the experiment, the animals were transferred from the animal facility to the experimental room at least $60 \mathrm{~min}$ before the start of the experiment. After measuring body weight, the mouse was placed in an open-field test apparatus to assess locomotor activity. The test apparatus surrounded a $60 \mathrm{~cm}$ diameter circular open-field figure 1a. Schematically shows the time schedule for each experiment. Each mouse was removed from its home cage and placed in the center of the apparatus. After a 90-min habituation period, the mouse was removed from the apparatus, intraperitoneally administrated with SA, COC5, COC10, COC20, ETH1, ETH2, ETH4, NIC2, NIC4, or NIC8 and returned to the apparatus for another $90 \mathrm{~min}$. We examined 10 mice for each drug (a total of 100 mice).

Experiments were performed out of the sight of the experimenter and a video camera (DCR-VX2100; Sony, Japan). Since the movement data of mice with a high resolution would be very large size in computer, a program on Linux is easy to use including the processing of output file. Therefore, we developed a program, glana, on Linux by ourselves with the $C$ language $[15,16]$. We measured the ordinates of the center of gravity from the target mouse images as $\vec{P}(t)=(x(t), y(t))$ with use of glanas. The program automatically computed the ordinates of center of gravity of mouse after discriminated the mouse image from the background with a high resolution (29.97 frames per second with a spatial resolution of approximately $0.25 \mathrm{~cm}$ per video pixel). The data of the ordinates of gravity center of mouse were processed with programs written by the Perl programming language [17].

During a so called stop state, a mouse may perform many local movements, such as rearing backward and stepping sideways; therefore, stop state does not necessarily imply zero speed. Drai et al. [7] developed the procedure to segment rodent's behavior into "stop state" or "moving state". Comparing the time evolution of walking speed in rodent's behavior (walking freely or sleeping), they found out that the inflexion point of estimated density function of walking speed could be interpreted as indicating the speed threshold to segment "stop state" or "moving state". This procedure was applied in the other papers [5-8].

Following the procedure developed by Drai et al. [7] described above, we measured the movement threshold as 0.8 pixels/time frame as shown in Figure 1b; values below this threshold were considered a stop state, and values above this threshold were considered a moving state. The moving duration represents the length of time in the moving state (gray area) in Figure 1c for each event of movement. The gaps between the moving durations represent the stop states.

\section{Data analysis}

Detecting the number of locomotor activity processes in the time scale: Initially introduced in statistical physics, log-log plots can reveal information regarding the distribution of walking movement [12-14]. It provides values for distinct processes of locomotor activity for different time scales from experimental trajectories $[13,14]$.

Position data of the mouse in the first 30 -min period of the habituation period (BF30M) and in the first 30-min period after drug administration (AF30M) were converted to sequential event data of the moving or stop state. Then, we counted the number of video frames for the moving duration, and the data for the 10 mice for each drug were summed. Plotting the moving duration data against the frequency of moving events on a log-log axis, we determined the appropriate number of processes by finding the best-fit model between Model 0: $\frac{\theta}{t^{\lambda}}$ , Model 1: $\theta_{1} \exp ^{-\lambda_{1} t}$, Model 2: $\sum_{i=1}^{2} \theta_{i} \exp ^{-\lambda_{t} t},(\mathrm{i}=1 ; 2), \ldots$, and Model N: $\sum_{i=1}^{N} \theta_{i} \exp ^{-\lambda t} ;(\mathrm{i}=1 ; \ldots, \mathrm{N})$, where the parameters $\lambda ; \theta ; \lambda_{\mathrm{i}} ; \theta_{\mathrm{i}}(\mathrm{i} ; 1 ; 2 ; \ldots, \mathrm{N})$ were estimated [15-17].
We utilized the model selection procedure developed by statistical theory $[18,19]$. As a criterion for selecting the best model, we adopted Akaikes information criterion (AIC) [18], which is a measure of the goodness of fit to the data of an estimated statistical model. AIC is defined as

$$
\mathrm{AIC}=-2 \ln \mathrm{L}+2 \mathrm{n},
$$

where $n$ is the number of parameters in the model and $L$ is the maximized value of the likelihood function for the estimated model de_ned below. Each statistical model is defined by parameter sets $\vec{\theta}=\left\{\theta_{1}, \theta_{2}, \ldots ., \theta_{n}\right\}$ and $\vec{\lambda}=\left\{\lambda_{1}, \lambda_{2}, . ., \lambda_{n}\right\}$. Let $\mathrm{z}(\mathrm{x} ; \vec{\theta} ; \vec{\lambda})$ be the distribution under a given parameter set. If the noise follows a normal distribution, the likelihood function is

$$
L\left(\vec{\theta}, \vec{\lambda}, \sigma^{2}\right)=\frac{1}{\left(2 \pi \sigma^{2}\right)^{N / 2}} \prod_{i=1}^{N} \exp \left[-\frac{\left\{y_{i}-Z(x ; \vec{\theta}, \vec{\lambda})\right\}^{2}}{2 \sigma^{2}}\right]
$$

where a pair $\left(y_{i}, x_{i}\right)$ is a data point of a histogram of the move-steplength distribution. $\sigma^{2}$ is the variance of data and is assumed to be independent of the position and time. $\mathrm{N}$ is the number of data.

Division of walking events into categories: If the moving duration distributions were expressed by the sum of exponential functions, we surmised that distinct processes might exist for different time scales. Because the time constants for each exponential mode of walking behavior of mice without drug administration might be intrinsic, we defined the decay times of each exponential function $\leq 1 / 10$ as the discrimination times.

\section{Statistical analysis}

The results were compared between the control (SA) and drug groups using 2-tailed Mann-Whitney U-tests. Statistical analyses were performed using SPSS 16.0 for Macintosh (SPSS Inc., Chicago, IL, USA).

\section{Results}

\section{The three-mode model is appropriate}

The log-log plot of the move-step frequency versus moving duration after SA, COC20, ETH4, and NIC8 administration are shown in Figure 2a-2d respectively.

The AIC values for the different statistical models are shown in Figure 2 and Table 1. We found that the model with the lowest AIC value (i.e. the best model) was Model 3, in which the composition of the three-exponential-model was the best to fit for the obtained distribution. The three-mode composition showed the best fit for not only the results shown in Table 1 but also the combined data for all the 10 drugs and almost all of the individual mouse data (data not shown). Therefore, we concluded that the moving-duration distributions involved 3 Poisson processes.

\section{Division of movement into three categories}

As the moving duration distributions were expressed by the sum of three exponential functions, we surmised that three distinct processes might exist for different time scales. If the fastest component of the exponential function decayed by $<1 / 10$ within seven video frames, the second component would decay within 70 video frames. Therefore, we classified all of the walking events into 3 categories according to the movement duration time: short-period movement (SPM) for 0-7 video-time-frame steps $(\leq 0.23 \mathrm{~s})$ medium period movement (MPM) for 8-70 steps $(0.27 \leq \mathrm{t} \leq 2.3 \mathrm{~s})$, and long-period movement (LPM) for 
Citation: Shoji H (2014) Physical Separation of Motion into Three Categories for Evaluating the Behavioral Effects of Drugs in Mice. Int $\mathrm{J}$ Neurorehabilitation 1: 120. doi:10.4172/2376-0281.1000120

(a)
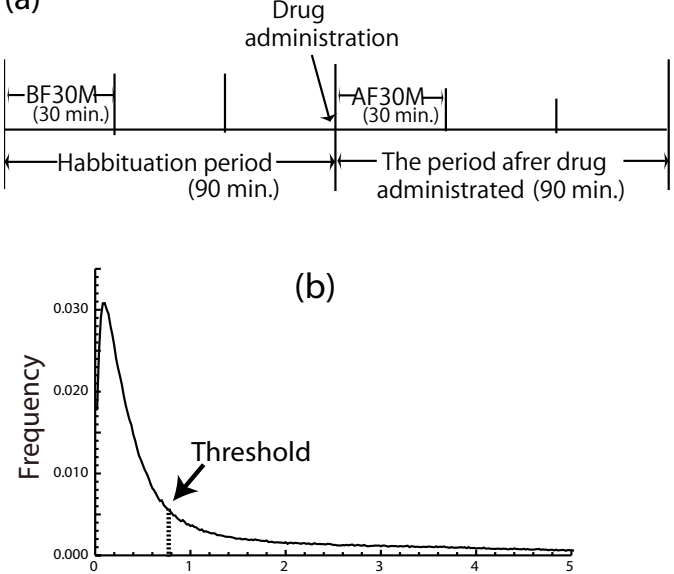

Pixels / (video Frame) (c)

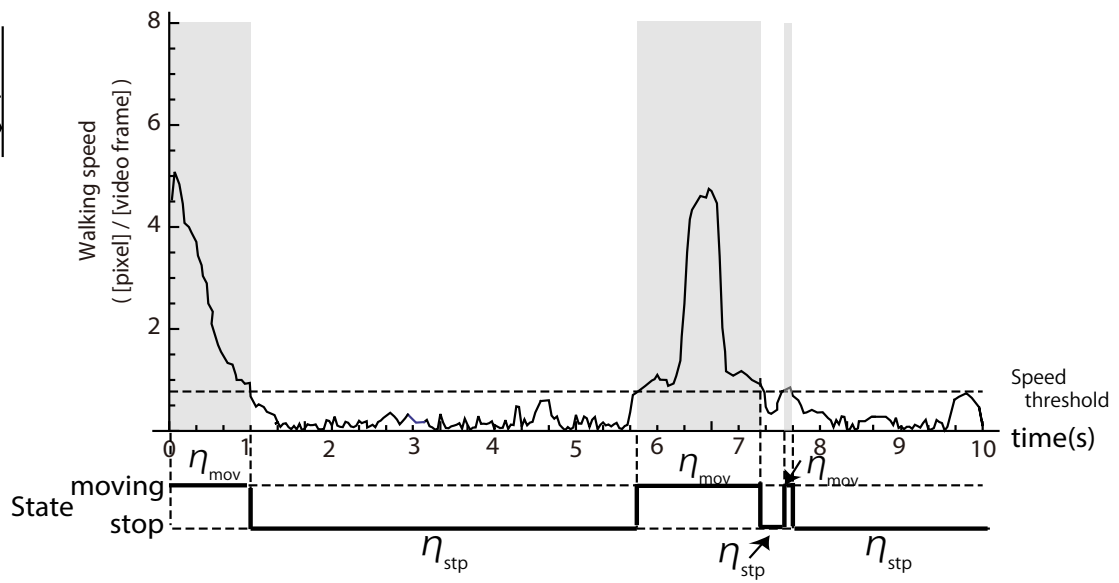

Figure 1: Principles of experimental procedures. (a) Time schedule of experiment for each mouse. We mainly performed the analysis in the first 30 -min period of habituation period (BF30M) and the first 30-min period after drug-administration (AF30M). (b) The frequency distribution of movement per $1 / 29.97$ sec. (video frame). Following the procedure developed by Drai et al. [7], the speed threshold to divide the moving or stop state is deter-mined at 0.8 pixels by the position of fixation point (dotted line) of the density estimated function indicated by the solid line. (c) A sample of the time series of a 10-s move-step length of a mouse administrated with COC20. Because of high-resolution video tracking, the speed and state changes are converted the scatter plot to line plot. Transformation of continuous variables, speed and position, into binary symbols. A speed threshold was set to differentiate the period of moving state (Gray) and of stop state(White).

(a)

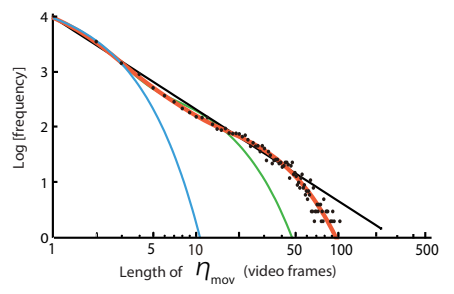

(b)

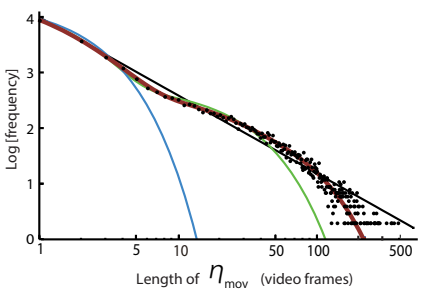

(c)

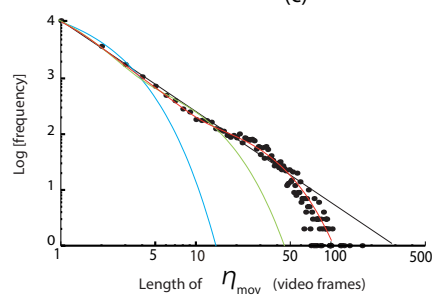

(d)

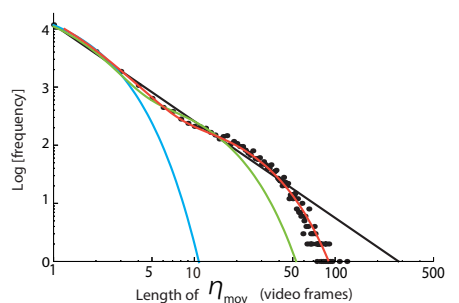

Figure 2: The log-log plots of moving duration versus their frequency for cumulative occurrence of moving events in AF30M of SA (a), COC20(b), ETH4(c), and NIC8(d) in mice. Dots indicate the obtained data. The black, blue, green and red lines indicate the fitted function using Model $0\left(\left(\theta / t^{\lambda}\right)\right)$, Model $1\left(\theta_{1} \exp (-\lambda t)\right)$, Model 2 $\left(\sum_{i=1}^{2} \theta_{i} \exp \left(-\lambda_{i} t\right)\right)$ and Model $33\left(\sum_{i=1}^{3} \theta_{i} \exp \left(-\lambda_{i} t\right)\right)$ respectively. The AIC values of each model are shown in Table 1 . Even if the number of considerable components $\theta_{i} \exp \left(-\lambda_{i} t\right)$ are increased, the Model 3 (solid red lines) are the best fitted in all the cases, according to the AIC criteria, as explained in the text and Table 5 .

\begin{tabular}{|c|c|c|c|c|c|c|c|c|}
\hline \multirow[b]{2}{*}{ Model } & \multicolumn{4}{|c|}{ After administration(AF30M) } & \multicolumn{4}{|c|}{ Before administration(BF30M) } \\
\hline & COC20 & ETH4 & NIC8 & SA & COC20 & ETH4 & NIC8 & SA \\
\hline $\operatorname{AIC}(0)$ & 3759.76 & 1438.16 & 1359.28 & 1136.95 & 1983.72 & 2253.81 & 2098.37 & 2407.30 \\
\hline $\operatorname{AIC}(1)$ & 4496.04 & 1607.93 & 1591.76 & 1242.74 & 1967.68 & 2382.96 & 2205.92 & 2533.76 \\
\hline $\operatorname{AIC}(2)$ & 3422.61 & 1285.27 & 1204.49 & 966.562 & 1637.95 & 2143.51 & 1913.59 & 2038.53 \\
\hline $\operatorname{AIC}(3)$ & 2704.17 & 1167.59 & 1057.36 & 854.432 & 1616.44 & 1967.35 & 1844.36 & 1811.40 \\
\hline $\mathrm{AIC}(4)$ & 2707.93 & 1171.63 & 1061.05 & 858.029 & 1620.15 & 1971.30 & 1848.51 & 1815.08 \\
\hline $\operatorname{AIC}(5)$ & 2711.38 & 1175.47 & 1065.29 & 861.829 & 1623.79 & 1975.24 & 1852.45 & 1817.49 \\
\hline Lowest & 3 & 3 & 3 & 3 & 3 & 3 & 3 & 3 \\
\hline 1 & 19046.1 & 44822.2 & 36359.6 & 29248.6 & 113348.0 & 54152.1 & 45046.7 & 122328.0 \\
\hline 2 & 5138.0 & 6424.6 & 9095.9 & 2317.7 & 3349.5 & 10248.2 & 9915.5 & 10767.1 \\
\hline 3 & 353.9 & 241.4 & 402.0 & 202.536 & 951.2 & 607.6 & 629.9 & 1364.1 \\
\hline 1 & 1.309 & 2.053 & 1.702 & 1.29 & 0.962 & 1.238 & 1.087 & 1.115 \\
\hline 2 & 0.481 & 0.567 & 0.643 & 0.394 & 0.203 & 0.352 & 0.331 & 0.362 \\
\hline 3 & 0.0363 & 0.0580 & 0.0679 & 0.0556 & 0.0482 & 0.0473 & 0.0522 & 0.0518 \\
\hline
\end{tabular}

Table 1: Summary of the AIC values for the log-log plot of th the moving duration and their frequency examined and the parameters in the tted function. COC20, ETH4, NIC8, and SA. 
Citation: Shoji H (2014) Physical Separation of Motion into Three Categories for Evaluating the Behavioral Effects of Drugs in Mice. Int $\mathrm{J}$ Neurorehabilitation 1: 120. doi:10.4172/2376-0281.1000120

Page 4 of 6

\begin{tabular}{|c|c|c|c|c|c|c|c|c|c|}
\hline & & BF30M & & & AF30M & & \multicolumn{3}{|c|}{ ratio(AF30M/BF30M) } \\
\hline & SPM & MPM & LPM & SPM & MPM & LPM & SPM & MPM & LPM \\
\hline SA & 12149 & 9505 & 167 & 4884 & 2996 & 74 & 0.401 & 0.315 & 0.443 \\
\hline coc5 & 12638 & 7798 & 168 & 5102 & 3502 & 147 & 0.404 & 0.449 & 0.875 \\
\hline $\mathrm{COC} 10$ & 14596 & 9648 & 186 & 6118 & 4214 & 266 & 0.419 & 0.437 & 1.430 \\
\hline COC20 & 10570 & 6830 & 176 & 6046 & 6455 & 1023 & 0.572 & 0.945 & 5.813 \\
\hline ETH1 & 12500 & 7395 & 150 & 6429 & 2534 & 32 & 0.514 & 0.343 & 0.213 \\
\hline ETH2 & 10946 & 6735 & 146 & 5943 & 2874 & 36 & 0.543 & 0.427 & 0.247 \\
\hline ETH4 & 11153 & 6424 & 160 & 8179 & 3286 & 54 & 0.733 & 0.512 & 0.338 \\
\hline $\mathrm{NIC2}$ & 14286 & 7200 & 141 & 14810 & 2720 & 5 & 1.037 & 0.378 & 0.0354 \\
\hline $\mathrm{NIC} 4$ & 15869 & 6659 & 143 & 11462 & 2958 & 17 & 0.722 & 0.444 & 0.119 \\
\hline NIC8 & 12082 & 6723 & 151 & 8444 & 3323 & 22 & 0.699 & 0.494 & 0.146 \\
\hline
\end{tabular}

Table 2: The total numbers of walking events of 10 individual during BF30M or AF30M session and their rations of the total numbers of events between AF30M and BF30M.

\begin{tabular}{|c|c|c|}
\hline Contrast & mode & P -values \\
\hline \multirow[t]{3}{*}{ SA vs. COC5 } & LPM & 0.00548 \\
\hline & MPM & 0.000908 \\
\hline & SPM & 0.000386 \\
\hline \multirow[t]{4}{*}{ SA vs. COC10 } & ALL & 0.0103 \\
\hline & LPM & 0.00949 \\
\hline & MPM & 0.000837 \\
\hline & SPM & 0.000124 \\
\hline \multirow[t]{3}{*}{ SA vs. COC20 } & ALL & 0.000161 \\
\hline & LPM & 0.0000761 \\
\hline & MPM & 0.000101 \\
\hline SA vs. ETH1 & SPM & 0.0450 \\
\hline SA vs. ETH2 & SPM & 0.0349 \\
\hline \multirow[t]{2}{*}{ SA vs. ETH4 } & SPM & 0.000606 \\
\hline & MPM & 0.00686 \\
\hline \multirow[t]{2}{*}{ SA vs. NIC2 } & MPM & 0.0432 \\
\hline & SPM & 0.000606 \\
\hline \multirow[t]{2}{*}{ SA vs. NIC4 } & MPM & 0.0349 \\
\hline & SPM & 0.00284 \\
\hline \multirow[t]{2}{*}{ SA vs. NIC8 } & MPM & 0.0393 \\
\hline & SPM & 0.0105 \\
\hline
\end{tabular}

Table 3: The statistical calculation for ratio of the total numbers of events during BF30M and AF30M comparing between controls (SA) and drugs (COC5, COC10, COC20, ETH1, ETH2, ETH4, NIC2, NIC4, or NIC8). Significance was set at P < 0:05. mode means walking categories, SPM, MPM, LPM, or ALL which is the case when the walking categories are not divided.
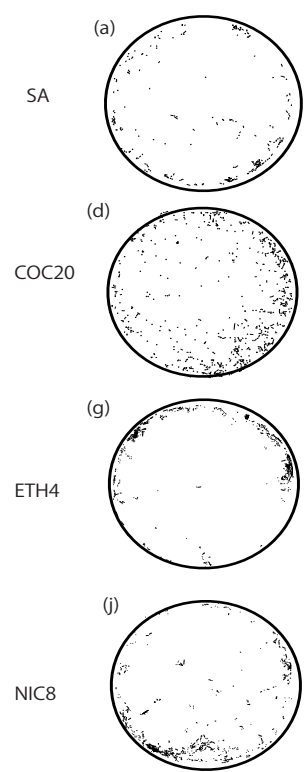

Figure 3: Trajectories of long-period movement (LPM) events (right), mediumperiod movement (MPM) event (middle), and short-period movement (SPM) events (left) during the first 30-min period after drug administration (AF30M) in the test apparatus. (a)-(c) control (SA), (d)-(f) COC20, (g)-(i) ETH4, and (j)-(I)NT8.

\begin{tabular}{|c|c|c|c|c|c|c|c|c|c|}
\hline & \multirow[b]{2}{*}{ SPM } & \multirow{2}{*}{$\begin{array}{l}\text { BF30M } \\
\text { MPM }\end{array}$} & \multirow[b]{2}{*}{ LPM } & \multirow[b]{2}{*}{ SPM } & \multirow{2}{*}{$\begin{array}{l}\text { AF30M } \\
\text { MPM }\end{array}$} & \multirow[b]{2}{*}{ LPM } & \multicolumn{3}{|c|}{ ratio(AF30M/BF30M) } \\
\hline & & & & & & & SPM & MPM & LPM \\
\hline SA & 288.54 & 1459.22 & 224.87 & 72.42 & 65.84 & 142.90 & 0.251 & 0.0451 & 0.635 \\
\hline coc5 & 172.46 & 1585.87 & 234.80 & 218.32 & 771.21 & 159.63 & 1.266 & 0.486 & 0.680 \\
\hline $\operatorname{coc} 10$ & 194.77 & 1760.39 & 239.74 & 583.70 & 1427.61 & 171.16 & 0.931 & 2.22 & 0.714 \\
\hline COC20 & 626.82 & 1459.22 & 224.87 & 677.60 & 487.70 & 46.34 & 1.081 & 0.758 & 0.450 \\
\hline ETH1 & 60.46 & 655.12 & 58.94 & 15.15 & 256.19 & 42.59 & 0.251 & 0.391 & 0.723 \\
\hline ETH2 & 56.19 & 637.56 & 48.59 & 19.88 & 222.82 & 33.19 & 0.354 & 0.349 & 0.683 \\
\hline ETH4 & 54.40 & 581.48 & 51.00 & 35.52 & 255.69 & 50.96 & 0.653 & 0.440 & 0.997 \\
\hline $\mathrm{NIC2}$ & 46.20 & 584.41 & 108.96 & 3.84 & 217.18 & 93.85 & 0.08317 & 0.372 & 0.861 \\
\hline $\mathrm{NIC} 4$ & 39.10 & 589.20 & 88.13 & 46.20 & 46.20 & 46.20 & 1.181 & 0.0784 & 0.524 \\
\hline NIC8 & 47.91 & 51.49 & 53.48 & 9.17 & 202.63 & 46.53 & 0.191 & 0.394 & 0.870 \\
\hline
\end{tabular}

Table 4: The total distance traveled in open eld of 10 mice for each drug administration during BF30M or AF30M sessions and their ratios between the distance during AF30M and BF30M. 
$>70$ steps $(>2.3 \mathrm{~s})$. After dividing moving event into three categories, the number of events in BF30M and AF30M were calculated as shown in Table II. The number of samples in LPM was greater with a higher COC dose.

Trajectories in AF30M of one individual sample of each drug (SA, COC20, ETH4, and NIC8) are shown in Figure 3. We found differences in the following two points, number of events, and walking event distances during walking events. Therefore, we examined the differences in these two points for each walking category (LPM, MPM, or SPM).

\section{Number of walking events}

We calculated the total number of walking events in each walking category during BF30M and AF30M. Table 2 shows the total number of walking events of 10 mice for each drug. The ratios of the number of events for each individual mouse were compared before and after drug administration. These were expected to be less affected by the variance of each individual experimental mouse.

Table 3 shows the statistical results. Significant differences were observed between experimental groups after separation into the 3 categories more often than when the moving events were not divided. We found the differences only in SA vs. COC10 and vs. COC20 when we did not divided the walking events into categories. However, after the division into three categories, we found the significant differences not only in these two contrasts but also another 7 contrasts as shown in Table 4.

\section{Walking distance}

We independently calculated the total distance freely traveled in the open field from the ordinate data during BF30M ( $\left.D_{i}^{\text {bf }}\right)$ and and $\operatorname{AF} 30 \mathrm{M}\left(D_{j}^{\text {bf }}\right)$ where $j(1 \leq j \leq 10)$ indicates the individual mouse number. Table 4 shows the total distance traveled of 10 mice for each drug. The ratios of the walking distances for each individual mouse before and after drug administration were examined; these were also expected to be less affected by the variance of each individual experimental mouse. There were significant differences in the $\mathrm{D}_{i}^{\text {af }}=\mathrm{D}_{j}^{\mathrm{bf}}$ ranges between the experimental groups. Table 5 shows the statistical results. Significant differences were observed between experimental groups after separation into the 3 categories more often than when the moving events were not divided.

\begin{tabular}{|c|c|c|}
\hline Contrast & mode & P -values \\
\hline & & 0.0235 \\
\hline SA vs. COC20 & ALL & 0.00682 \\
\hline & LPM & 0.0227 \\
\hline SA vs. COC10 & MPM & 0.0447 \\
\hline & LPM & 0.0389 \\
\hline SA vs. COC5 & MPM & 0.0401 \\
\hline & MPM & \\
\hline SA vs. ETH1 & LPM & 0.0278 \\
\hline & & 0.0449 \\
\hline SA vs. NIC4 & LPM & 0.0490 \\
\hline SA vs. NIC2 & LPM & \\
\hline
\end{tabular}

Table 5: The statistical calculation for ratio of the ratio of the total distance freely trav-eled during BF30M and $A F 30 M\left(D_{i}^{\text {af }}=D_{i}^{\text {bf }}\right)$ comparing between controls $(S A)$ and drugs (COC5, COC10, COC20, ETH1, ETH2, ETH4, NIC2, NIC4, or NIC8). Significance was set at $\mathrm{P}<0.05$. mode means walking categories, SPM, MPM LPM, or ALL which is the case when the walking categories are not divided.

\section{Discussion}

In this study, we explored locomotor activity in drug-administered mice using the characterizations developed in physics. All the log$\log$ plots of moving duration vs. their frequency for the cumulative occurrence of the walking events for 10 individual mice shown in Figure 2 were best fit to the three-exponential-model, and almost of individual walking events (data not shown) were also fit best to the three-exponential-model. Therefore, walking movements were separated into three categories depending on walking durations, comprising the main approach described in this paper.

The differences in behavior after the drug administration were observed more often after the separation of walking events as shown in Table 3 and 5. Although we were unable to reveal the reasons why the index differences were observed more often after separating the walking event into categories, it might be useful to assess the differences between processes with different time scales. Although we divided that all the episodes depending on the moving duration, Drai et al. [7] and Kafkafi et al. [8] divided ones depending on the range of maximum speed to attain during each walking episode. They had showed the distributions could be typically expressed by three Gaussian modes using the Gaussian mixture model (GMM) [19] and the expectationmaximization (EM) algorithm [19]. We calculated on the maximam speeds during walking episodes in each group. The maximum ones in SPM, MPM and LPM were $0.94-23.9 \times 10^{3} \mathrm{~m} / \mathrm{s}$ in SPM, $2.95 \times 10^{3} \mathrm{~m} / \mathrm{s}$ in MPM, and $4.2-41.6 \times 10^{3} \mathrm{~m} / \mathrm{s}$ in LPM, respectively. These two different grouping may have the relations, which we study further in the future work.

Although significant differences in behavioral effects based on the stop-duration time have been reported in several previous studies $[12,20]$, we did not refer to stop-duration time in this paper because we did not obtain a consistent result for the best-fit distribution. The best fit included the five-exponential-mode model once and also the fourexponential-mode model once. Therefore, we did not perform further analysis. Paulus and Geyer $[10,11]$ also examined the log-log plots of moving duration versus their frequency for cumulative occurrence of moving events of drug administrated mice. Without any statistical comparisons, they concluded that the moving-duration distributions could be fit best by model 0 (power law distribution) by appearance. In their paradigm, the moving episodes could not divide into groups. While, we conducted the analysis by using a high resolution image of mice movements video, and we applied the AIC statistical approach to decide that 3-mode-model was the best fit. Moreover, an advantage of our paradigm described in this paper is that the difference was effectively detected after the episodes were separated into the 3 groups. For example, the frequency of LPM episodes of COC shown in Table 5 was far lesser than the others. Therefore, it might be useful to assess the differences in COC effects between processes with different time scales. We previously developed the index to differentiate dose-related behavioral effects of COC, concentrating on LPM walking category events [16]. Because the LPM walking events occurred the least frequently, it was difficult to detect a difference without concentrating solely on LPM walking events. Therefore, separation depending on moving duration might ultimately help to differentiate the behavioral effects of drugs, and we hope to broaden our understanding of complex behavioral traits using this procedure.

\section{Acknowledgment}

We thank K. Hanai and C. Yokoyama for their technical assistance and helpful 
Citation: Shoji H (2014) Physical Separation of Motion into Three Categories for Evaluating the Behavioral Effects of Drugs in Mice. Int $\mathrm{J}$ Neurorehabilitation 1: 120. doi:10.4172/2376-0281.1000120

comments. This study was supported in part by a Grant-in-Aid of Japan Society for the Promotion of Science (KAKENHI 26800225).

\section{References}

1. Carlson NR (2004) Physiology of behavior, 8th edition. Pearson International Edition, Boston.

2. Kafkafi N, Elmer G (2005) Activity density in the open field : a measure for differentiating the e ect of psychostimulants. Phamcol Biochem Behav 80: 239249

3. Simon P, Dupuis R, Costentin J (1994) Thigmotaxis as an index of anxiety in mice. In ence of dopaminergic transmissions. Behav Brain Res 61: 59-64.

4. Lipkind D, Sakov A, Kafka N, Elmer GI, Benjamini Y, et al. (2004) New replicable anxiety-related measures of wall vs. center behavior of mice in the open field. J Appl Physiol 97: 347-359.

5. Benjamini Y, Lipkind D, Horev G, Kafka N, Golani I, et al. (2010) Ten ways to improve the quality of descriptions of whole-animal movement. Neurosc Biobehav Rev 34: 1351-1365.

6. Kafkafi N, Lipkind D, Benjamini Y, Mayo CL, Elmer GI, et al. (2003) SEE locomotor behavior test discriminates C57BL/6J and DBA/2J mouse inbred strains across laboratories and protocol conditions. Behav Neurosci 117: 464477.

7. Drai D, Benjamini Y, Golani I (2000) Statistical discrimination of natural modes of motion in rat exploratory behavior. J Neuroscience Methods 96: 119-131.

8. Kafkafi N, Mayo C, Drai D, Golani I, Elmer G (2001) Natural segmentation of the locomotor behavior of drug-induced rats in a pho-tobeam cage. J Neuroscience Method 109: 111-121.

9. Paulus MP, Geyer MA, Gold LH, Mandell AJ (1990) Application of entropy measures derived from the ergodic theory of dy-namical systems to rat locomotor behavior. Proc Nat Acad Sci USA 87: 723-727.

10. Paulus MP, Geyer MA (1991) A temporal and spatial scaling hypothesis for the behavioral e ects of psychostimulants, Psychopharmacology 104: 6-16.

11. Paulus MP, Geyer MA (1993) Three independent factors characterize spontaneous rat motor activity. Behav Brain Res 53: 11-20.

12. Jensen HJ (1998) Self-Organized Criticality, Cambridge University Press 1998.

13. Barabasi AL (2005) Origin of burst and heavy tails in human dynamics. Nature 435: $207-211$.

14. Matthaus F, Mommer MS, Curk T, Dobnikar J (2011) On the origin and characteristics of noise-induced Levy walks of E. coli. PLos ONE 6: e18623.

15. Hanai K, Ozaki M, Yamauchi D, Nakatomi Y, Yokoyama C, et al. (2006) Scale free dynamics involved in locomotor activity of ant and mouse. WSEAS Trans Biol Biomes 6: 511-515.

16. Shoji H, Nakatomi Y, Yokoyama C, Fukui K, Hanai KJ (2013) New index based on the physical separation of motion into three categories for characterizing the effect of cocaine in mice. Theor Biol 333: 68-77.

17. Wall L, Christiansen T, Orwant J (2000) Programming Perl, 3rd Edition. O'Reilly Media.

18. Akaike H (1974) A new look at statistical model identification. IEEE Trans Automat Control 19: 716-723.

19. Bishop CM (2006) Pattern Recognition and Machine Learning. New York: Springer-Verlag.

20. Viswanathan GM, Afanasyeev V, Buldyrev SV, Murphy EJ, Price PA, et al. (1996) Levy flight search patterns of wandering albatrosses Nature 381: 413- 